\section{SAT0212 OBSTETRIC AND THROMBOTIC ANTIPHOSPHOLIPID SYNDROME: SIMILAR ANTIBODIES BUT DIFFERENT PHENOTYPES?}

H. Jiang ${ }^{1}$, Y. Zhao ${ }^{1}$, C. H. Wang ${ }^{1}$, J. Zhao ${ }^{1} .{ }^{1}$ Peking Union Medical College Hospital, Rheumatology, Beijing, China

Background: Several studies showed two main clinical phenotypes of antiphospholipid syndrome which could be independent, but only a few data contrast features between these two groups.

Objectives: To investigate whether obstetric and thrombotic manifestations of APS are independent subtypes.

Methods: This was a single center prospective study from the PUMCH database of primary antiphospholipid syndrome followed for over 4years. Comparing demographic data, laboratory tests, pregnancy morbidity and thrombotic events during follow-up between loAPS (isolated obstetric APS) and ItAPS (isolated thrombotic APS).

Results: A total of 244 patients was registered in $\mathrm{PUMCH}$ primary APS cohorts, $157(64.34 \%)$ were female patients. In female patients, $44(28.03 \%)$ were diagnosed with IoAPS, $42(26.75 \%)$ were ItAPS. Demography showed patients in ItAPS group were older than loAPS group (40 vs $33, p<0.001$ ), presented more cardiovascular risks $(33.33 \%$ vs $6.8 \%, p<0.01)$, neurological disorders $(23.8 \%$ vs $2.3 \%, p<0.01)$ and thrombocytopenia $(47.6 \%$ vs $20.5 \%, p<0.01)$. Antibody profiles had no difference in triple positivity, double positivity and partial single positivity (ACL, LA), but presence of single anti- 32 GPI positivity showed significant difference between loAPS and ItAPS $(59.09 \%$ vs $38.1 \%, p<0.05)$. Significant difference was presented in homocysteine(Median) between loAPS and ItAPS ( 9.9 vs $11.5, \mathrm{p}<0.05$ ), not in inflammatory markers. During 49.5 (Median) months follow-up of ItAPS group, patients got 90 pregnancies, 5 abortions but weren't fulfilled with the diagnosis criteria of pregnancy morbidity. No thrombotic event occurred during 48.5(Median) months follow-up time in loAPS group.

Conclusion: IoAPS and ItAPS shared similar antibody profile, but presented isolated clinical complications, different demographic features and maintained independent manifestation during follow-up, indicating the underlying pathogeneses are different.

Table1. Demographic characteristics

\begin{tabular}{lccc}
\hline & IoAPS $(\mathrm{n}=44)$ & ItAPS $(\mathrm{n}=42)$ & P-value \\
\hline Age, years, Median (Q1-Q3) & $33(30.00,36.00)$ & $40(33.75,55.25)$ & .00 \\
B.M.I. Median (Q1-Q3) & $22.86(20.70,24.45)$ & $23.52(21.44,27.70)$ & .10 \\
Flow-up time, month, & $48.50(36.00,77.00)$ & $49.50(23.00,103.75)$ & .44 \\
Median (Q1-Q3) & & & \\
Smoke (n\%) & $1(2.27)$ & $0(0)$ & 1.00 \\
Cardiovascular risk factors (n\%) & $3(6.82)$ & $14(33.33)$ & .00 \\
Coronary heart disease & $0(0)$ & $2(4.76)$ & .24 \\
Hypertension & $1(2.27)$ & $5(11.90)$ & .11 \\
Obesity & $2(4.55)$ & $9(21.43)$ & .03 \\
Diabetes mellitus & $0(0)$ & $0(0)$ & \\
Complications (n\%) & $16(36.36)$ & $30(71.43)$ & .00 \\
Kidney diseases & $2(4.55)$ & $3(7.14)$ & .67 \\
Thrombocytopenia & $9(20.45)$ & $20(47.62)$ & .01 \\
Neurological Disorders & $1(2.27)$ & $10(23.81)$ & .00 \\
Stroke & $0(0)$ & $8(19.05)$ & .00 \\
Non stroke CNS manifestations & $1(2.27)$ & $2(4.76)$ & .61 \\
Valvular heart diseases & $4(9.09)$ & $2(4.76)$ & .68 \\
Failed pregnancy during follow-up (\%) & $116 / 154(75.32)$ & $5 / 90(5.56)$ & .00 \\
Thrombosis during follow-up (n\%) & $0(0)$ & $4(9.52)$ & .05 \\
\hline
\end{tabular}

Table 2. Laboratory tests

\begin{tabular}{|c|c|c|c|}
\hline & IoAPS $(n=44)$ & ItAPS(n=42) & P-value \\
\hline \multicolumn{4}{|l|}{ Antibody categories } \\
\hline Triple positive (n\%) & $11(25.00)$ & $15(35.71)$ & .35 \\
\hline \multicolumn{4}{|l|}{$A C L+\beta 2 G P I+L A$} \\
\hline Double positive (n\%) & $7(15.90)$ & $11(26.19)$ & .29 \\
\hline $\mathrm{LA}+\mathrm{ACL}$ & $0(0)$ & $1(2.38)$ & .49 \\
\hline LA+anti- $\beta 2$ GPI & $2(4.55)$ & $3(7.14)$ & .67 \\
\hline ACL+anti- $\beta 2 \mathrm{GPI}$ & $5(11.36)$ & $7(16.67)$ & .55 \\
\hline Single positive ( $n \%)$ & $26(59.10)$ & $16(38.10)$ & .04 \\
\hline $\mathrm{ACL}$ & $1(2.27)$ & $1(2.38)$ & 1.00 \\
\hline anti- $\beta 2$ GPI & $23(52.27)$ & $10(23.81)$ & .01 \\
\hline LA & $2(4.55)$ & $5(11.90)$ & .26 \\
\hline Negative after treatment & $4(9.09)$ & $1(2.38)$ & .36 \\
\hline ESR & $10(6,27)$ & $9(4,16)$ & .06 \\
\hline CRP & $1.03(0.56,3.85)$ & $1.11(0.43,3.18)$ & .63 \\
\hline $\mathrm{HCY}$ & $9.9(8.10,12.10)$ & $11.5(9.30,14.65)$ & .04 \\
\hline
\end{tabular}

* aCL: anticardiolipin antibodies; anti-ß2-GPI: anti- $\beta 2$ glycoprotein I antibodies; LA: lupus anticoagulant; ESR: erythrocyte sedimentation rate; CRP: C-reactive protein; HCY: homocysteine.

\section{References:}

[1] Meroni P L, Borghi M O, Grossi C, et al. Obstetric and vascular antiphospholipid syndrome: same antibodies but different diseases?[J]. Nat Rev Rheumatol, 2018, 14(7): 433-440

Acknowledgments: The authors thank Department of Rheumatology in Peking Union Medical College Hospital for support with statistics.

Disclosure of Interests: None declared

DOI: 10.1136/annrheumdis-2020-eular.5251

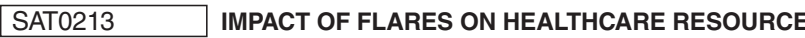 USAGE AND PROS IN SYSTEMIC LUPUS ERYTHEMATOSUS PATIENTS}

Z. Touma ${ }^{1}$, B. Hoskin ${ }^{2}$, C. Atkinson ${ }^{2}$, D. Bell ${ }^{2}$, O. Massey ${ }^{2}$, J. H. Lofland ${ }^{3}$, P. Berry ${ }^{3}$, C. Karyekar ${ }^{3}$, K. Costenbader ${ }^{4}{ }^{1}$ University of Toronto, Toronto, Canada; ${ }^{2}$ Adelphi Real World, Cheshire, United Kingdom; ${ }^{3}$ Global Commercial Strategic Organisation, Johnson and Johnson, Horsham, United States of America;

${ }^{4}$ Brigham and Women's Hospital, Boston, United States of America

Background: The effect of flares on healthcare resource usage and patient-reported outcome scores in SLE patients is not well quantified.

Objectives: To understand how flares impact healthcare resource utilization (HCRU) and patient-reported outcomes amongst an international real-world dataset of SLE patients.

Methods: The Adelphi Real World 2015 Lupus Disease Specific Programme (DSP) is a cross-sectional study of 263 rheumatologists in the US and EU5. Rheumatologists were asked to complete patient record forms (PRFs) for the next 5 prospectively consulting SLE patients; the same patients were asked to complete patient self-completion (PSC) forms describing how SLE affected them PRFs collected data pertaining to the patient's diagnosis, disease history, current clinical outcomes, treatment and management history. PSCs collected similar data and included patient-reported outcome measures (PROs) to assess humanistic burden. Propensity score matching was used to assess differences in HCRU and health status between SLE patients who had flared (physician defined) in the last 12 months and those who had not. Matching variables were patient ethnicity, time since diagnosis, and severity at diagnosis. Data were extracted from 1278 PRFs, and 591 PSCs. Propensity score matching was carried out on two matched groups of 408 patients.

Results: Demographic data are reported in Table 1. Propensity score matching showed patients who flared in the last 12 months experienced significantly greate hospitalizations, visits to the ER, and total HCP consults in the last 12 months. Significantly greater drug burden lower physician and patient satisfaction, lowe EQ-5D score (worse health status), lower FACIT Fatigue score (greater fatigue), and greater overall work impairment (Table 2) were also observed.

\section{Table 1. Demographic data}

\begin{tabular}{lcc} 
Variable & Flared in last $\mathbf{1 2}$ months & Not flared in the last 12 months \\
\hline Mean age (years) & 41.8 & 42.4 \\
\% Female & 86.0 & 87.0 \\
\% White/Caucasian & 66.2 & 76.3 \\
Mean years diagnosed & 5.9 & 5.4 \\
\hline
\end{tabular}

Table 2. Propensity score matching results

\begin{tabular}{|c|c|c|c|c|c|}
\hline Outcome variable & $\begin{array}{l}\text { Flared } \\
\text { mean }\end{array}$ & $\begin{array}{l}\text { Not flared } \\
\text { mean }\end{array}$ & Coefficient & $95 \% \mathrm{Cl}$ & p-value \\
\hline Hospitalisations in last 12 months & 24.26 & 7.63 & 0.17 & {$[0.12-0.21]$} & ] $<0.001$ \\
\hline $\begin{array}{l}\text { Emergency department visits in last } \\
12 \text { months }\end{array}$ & 20.83 & 4.19 & 0.17 & {$[0.12-0.21]$} & $<0.001$ \\
\hline Number of tests in last 12 months & 46.49 & 38.90 & 7.59 & {$[3.74-11.44]$} & $<0.001$ \\
\hline Number of current medications & 2.76 & 2.19 & 0.57 & {$[0.43-0.72]$} & $<0.001$ \\
\hline Physician satisfied & 64.46 & 86.63 & -0.22 & $\begin{array}{l}{[-0.28} \\
--0.17]\end{array}$ & $<0.001$ \\
\hline Patient satisfied & 69.29 & 85.09 & -0.16 & $\begin{array}{l}{[-0.24} \\
--0.08]\end{array}$ & $<0.001$ \\
\hline EQ-5D-3L & 0.72 & 0.83 & -0.11 & $\begin{array}{l}{[-0.15} \\
--0.07]\end{array}$ & $<0.001$ \\
\hline FACIT Fatigue & 30.06 & 36.48 & -6.42 & {$[-8.5--4.3]$} & $<0.001$ \\
\hline $\begin{array}{l}\text { WPAI overall percentage work } \\
\text { impairment }\end{array}$ & 42.74 & 30.23 & 12.5 & {$[7.51-17.50]$} & ] $<0.001$ \\
\hline
\end{tabular}

Conclusion: The analysis of international real-world data confirmed that SLE patients who flared in the last year represent a greater burden on healthcare resource and demonstrate significantly worse health status, greater fatigue, lower 
patient and physician satisfaction and greater overall work impairment compared with non-flaring patients. There is a need for more effective treatments in this patient population to reduce patient and healthcare burden.

Study funded by Johnson and Johnson.

Disclosure of Interests: Zahi Touma Consultant of: Consultant for Janssen, Ben Hoskin Consultant of: Consultant for Janssen, Christian Atkinson Consultant of: Consultant for Janssen, David Bell Consultant of: Janssen, Olivia Massey Consultant of: Janssen, Jennifer H. Lofland Employee of: Janssen, Pamela Berry Employee of: Janssen, Chetan Karyekar Shareholder of: Johnson \& Johnson, Consultant of: Janssen, Employee of: Janssen Global Services, LLC. Previously, Novartis, Bristol-Myers Squibb, and Abbott Labs., Karen Costenbader Grant/ research support from: Merck, Consultant of: Astra-Zeneca

DOI: 10.1136/annrheumdis-2020-eular.6010

\section{SAT0214 ULTRASONOGRAPHIC CHANGES OF SALIVARY GLANDS IN PRIMARY SJOGREN'S SYNDROME: A LONGITUDINAL PROSPECTIVE STUDY}

H. R. Kim ${ }^{1}$, K. A. Lee ${ }^{2}$, S. H. Lee ${ }^{1}$, S. H. Kim ${ }^{1} .{ }^{1}$ Konkuk University School of Medicine, Seoul, Korea, Rep. of (South Korea); ${ }^{2}$ Soonchunhyang University Seoul Hospital, Seoul, Korea, Rep. of (South Korea)

Background: In the diagnosis of primary Sjogren' syndrome (SS), salivary gland ultrasound is useful tool. Until now, there is no data for ultasonographic changes of major salivary glands over time.

Objectives: This study aimed to evaluate the changes in abnormalities of salivary gland ultrasound (SGUS) over time in patients with pSS.

Methods: Patients with pSS $(n=70)$ and idiopathic sicca syndrome $(n=18)$ underwent SGUS twice at baseline and 2 years later. The semi-quantitative SGUS score (0-48) was used, which comprises five parameters: parenchymal echogenicity, homogeneity, hypoechoic areas, hyperechogenic reflections, and clearness of posterior borders. The intraglandular power Doppler signal (PDS) was also assessed. The changes of these SGUS variables were compared in patients with pSS and idiopathic sicca syndrome.

Results: The median (interquartile range) total SGUS scores at baseline was 27 (14) in patients with and 4 (3) in those with idiopathic sicca syndrome $(p<0.001)$. In the pSS group, the total SGUS scores and the SGUS scores for bilateral parotid glands were significantly increased during median 23.4 month follow-up ( $p=0.013$ and $p=0.011$, respectively). Homogeneity and hypoechoic areas were the domain to show statistically significant progression of SGUS scores. None of the SGUS scores changed significantly in the patients with idiopathic sicca syndrome. In patients with pSS, baseline and follow-up PDS sum scores of four salivary glands were significant higher in worsening SGUS group $(n=13)$ than no change/improvement SGUS group $(n=55 / 2)$.

Conclusion: The structural abnormalities in major salivary glands assessed using SGUS scores progressed significantly in patients with pSS. In pSS group, $18.6 \%$ patients had worsening SGUS scores during 2 years. Intra-glandular hypervascularity was associated with worsening of salivary gland abnormalities. References:

[1] Delli K, Dijkstra PU, Stel AJ, Bootsma H, Vissink A, Spijkervet FK. Diagnostic properties of ultrasound of major salivary glands in Sjogren's syndrome: a meta-analysis. Oral diseases. 2015;21(6):792-800.

[2] Jousse-Joulin S, Devauchelle-Pensec V, Cornec D, Marhadour T, Bressollette L, Gestin S, et al. Brief Report: Ultrasonographic Assessment of Salivary Gland Response to Rituximab in Primary Sjogren's Syndrome. Arthritis \& rheumatology (Hoboken, NJ). 2015;67(6):1623-8.

[3] Gazeau P, Cornec D, Jousse-Joulin S, Guellec D, Saraux A, Devauchelle-Pensec V. Time-course of ultrasound abnormalities of major salivary glands in suspected Sjogren's syndrome. Joint, bone, spine: revue du rhumatisme. 2018;85(2):227-32.

[4] Lee KA, Lee SH, Kim HR. Diagnostic and predictive evaluation using salivary gland ultrasonography in primary Sjogren's syndrome. Clinical and experimental rheumatology. 2018;36 Suppl 112(3):165-72.

Acknowledgments: : This work was funded by the Konkuk University Medical Center Research Grant 2019.

Disclosure of Interests: None declared

DOI: 10.1136/annrheumdis-2020-eular.337

\section{SAT0215 \\ HISTORY OF TONSILLECTOMY IS ASSOCIATED WITH GLANDULAR INFLAMMATION IN SJÖGREN'S SYNDROME}

F. Kollert $^{1,2}$, V. Pucino ${ }^{2,3}$, S. Rauz ${ }^{2,4}$, A. Richard ${ }^{5}$, J. Higham ${ }^{5}$, A. PovedoGallego $^{2,5}$, R. M. Brown ${ }^{6}$, T. Bates ${ }^{6}$, S. J. Bowman ${ }^{2,3}$, F. Barone ${ }^{2,3}$, B. Fisher ${ }^{2,3}$. ${ }^{1}$ Department of Rheumatology, Immunology and Allergology, Inselspital, University Hospital Bern, Bern, Switzerland; ' Institute of Inflammation and Aeging, College of Medical and Dental Sciences, University of Birmingham, Birmingham, United Kingdom; ${ }^{3}$ National Institute for Health Research (NIHR),
Birmingham Biomedical Research Centre and Department of Rheumatology, University Hospitals Birmingham NHS Foundation Trust, Birmingham, United Kingdom; ${ }^{4}$ Academic Unit of Opthalmology, Birmingham and Midland Eye Centre, Birmingham, United Kingdom; ${ }^{5}$ Department of Oral Medicine, Birmingham Dental Hospital, Birmingham, United Kingdom; ${ }^{6}$ Department of Histopathology, University Hospitals Birmingham NHS Foundation Trust, Birmingham, United Kingdom

Background: The palatine tonsils are secondary lymphoid-organs that serve as the first line of defense against pathogens. Whether history of tonsillectomy (TE) is associated with the phenotype of Sjögren`s syndrome (SjS) has not been investigated to date.

Objectives: To test whether TE is linked to SjS phenotype and disease activity scores.

Methods: A total of 183 patients from the Optimising Assessment in Sjögren`s Syndrome (OASIS) cohort with SjS or non-SjS sicca syndrome were analysed. Patients with SjS fulfilled 2016 ACR/EULAR classification for primary SjS; sicca patients had objective and/or subjective dryness, but were anti-Ro/SSA negative and had no physician diagnosis of SjS. One SjS patient who had TE around the time of symptom onset was excluded.

Results: Of the total cohort, 116 were diagnosed with SjS (86.2\% SSA/Ro positive) and 67 with non-SjS sicca syndrome. Overall, 29\% (53/183) had TE; $24.1 \%$ of the SjS patients $(28 / 116)$ and $37.3 \%$ of the sicca patients $(25 / 67)$. The prevalence of TE was higher in sicca than in SjS $(p=0.043)$. The median age at TE was 8 (range 3-50) years and did not differ between SjS and sicca patients $(p=0.629)$ Neither age at first symptoms $(p=0.093)$ nor disease duration $(p=0.623)$ were associated with TE in patients with SjS. SjS patients with TE showed a higher average histological focus score $(2.1(1.2-2.8)$ vs. $1.3(0.0-4.3) ; p=0.049)$, and were more likely to have activity in the glandular ( 53.6 vs. $20.5 \% ; p=0.001$ ) and constitutional ( 39.3 vs. $14.9 \%, p=0.014$ ) domains of the ESSDAI, and lower levels of IgG (12.2 (7.8-35.6) vs. $15.6(5.7-56.4) \mathrm{g} / \mathrm{l} ; \mathrm{p}=0.012)$ and $\lg \mathrm{A}(2.3(0.9-6.6)$ vs. $2.9(0.7-9.4) \mathrm{g} / \mathrm{l} ; \mathrm{p}=0.032)$. Whereas there was no difference in EQ5D utility values $(p=0.718)$, VAS global health was significantly lower in the patients with SjS who had TE (58 (10-78) vs. 70 (10-97); $p=0.021)$. There was no association between the status of TE and autoantibodies (SSA, SSB, RF), lachrymal and salivary glands function (Schirmer's test, unstimulated saliva flow), complement (C3, C4), serum levels of free light chains, $\beta 2$-microglobulin, ESSPRI, or total ESSDAI (all p-values >0.1). Of 181 patients, $12.7 \%(23 / 283)$ had appendectomy $(A E) ; 10.5 \%(12 / 114)$ of the SjS patients and $16.4 \%(11 / 67)$ of the sicca patients $(p=0.258)$. With the exception of lower unstimulated salivary flow $(0.086(0.01-$ $0.43)$ vs. $0.11(0.0-1.3) \mathrm{ml} / \mathrm{min} ; \mathrm{p}=0.026)$ in $\mathrm{SjS}$ patients with $A E$, there were no differences in disease phenotype between $\mathrm{SjS}$ patients with and without $A E$ (all p-values $>0.1$ ).

Conclusion: History of TE in SjS is associated with higher average focus scores and with glandular swelling. It could be speculated that the absence of palatine tonsils is compensated by enhanced lymphocytic infiltrates in the salivary glands. Further research is required to determine if TE is a risk factor for both $\mathrm{SjS}$ and non-SjS sicca and to determine the role of the tonsils in the generation of hypergammaglobulinaemia in SjS.

Disclosure of Interests: Florian Kollert Employee of: Novartis, Valentina Pucino: None declared, Saaeha Rauz: None declared, Andrea Richard: None declared, Jon Higham: None declared, Ana Povedo-Gallego: None declared, Rachel M. Brown: None declared, Timothy Bates: None declared, Simon J. Bowman Consultant of: Astrazeneca, Biogen, BMS, Celgene, Medimmune, MTPharma, Novartis, Ono, UCB, xtlbio, Glapagos, Speakers bureau: Novartis, Francesca Barone: None declared, Benjamin Fisher: None declared

DOI: 10.1136/annrheumdis-2020-eular.2112

\section{SAT0216 DISEASE SEVERITY, COMORBID CONDITIONS, TREATMENT PATTERNS, AND FLARES IN ADULTS WITH SYSTEMIC LUPUS ERYTHEMATOSUS IN THE UNITED KINGDOM: A REAL-WORLD OBSERVATIONAL RETROSPECTIVE COHORT ANALYSIS}

J. Langham ${ }^{1}$, V. Barut ${ }^{2}$, M. Samnaliev ${ }^{1}$, S. Langham ${ }^{1}$, S. Weir ${ }^{1}$, X. Wang ${ }^{3}$, B. Desta4, E. R. Hammond4. ${ }^{1}$ Maverex Limited, Manchester, United Kingdom; ${ }^{2}$ BioPharmaceuticals Medical, AstraZeneca, Cambridge, United Kingdom; ${ }^{3}$ BioPharmaceuticals Medical, AstraZeneca, Gaithersburg, United States of America; ${ }^{3}$ BioPharmaceuticals Medical, AstraZeneca, Gaithersburg, United States of America

Background: There is limited real-world evidence describing the presentation and treatment patterns of systemic lupus erythematosus (SLE) in the United Kingdom (UK).

Objectives: To characterize disease severity, comorbid conditions, treatment patterns, and flares in a longitudinal cohort of adults with SLE in the UK. 